\title{
Dancing with Isometries in Architecture
}

\author{
Arzu Gönenç Sorguç ${ }^{1}$
}

Published online: 20 May 2015

(C) Kim Williams Books, Turin 2015

\begin{abstract}
This paper aims to emphasize the role of mathematics in computational designeducation. A computational design process that forces designers to design not only theend product but also the design process itself requires a mind shift to enable thedesigner to develop algorithms and skills to deal with complex relations. In this context, understanding rule-based systems, generative systems, parametric models andcorresponding dimensionalities responding to the forces, variables, patterns, and themathematics behind them, becomes crucial. Illustrating the reciprocal relationshipbetween mathematics and architecture pattern studies offers great potentials. In thispaper, a series of explorations have been presented. In this exploration dance acts asa medium of inquiry into how different complexities can be mapped, how rules can begenerative (as first introduced in patterns) and how a set of rules can be transcodedinto a complex domain.
\end{abstract}

\section{Introduction}

The close relationship between mathematics and architecture can be observed starting from the early examples in the past to the present, still evolving/transforming each other reciprocally. When the definition of mathematics is considered, it can be seen that mathematics is not just a discipline studying numbers, quantities, relations, but also space, spatial relations, abstractions, models, transformations and more, through various branches of mathematics. Hence mathematics is beyond a field of science, it is a way of thinking, a language. Similarly, architecture cannot be considered as just the designing of buildings but as a discipline exploring space,

Arzu Gönenç Sorguç

arzug@metu.edu.tr; arzugonencsorguc@yahoo.com;

http://www.metu.edu.tr/ arzug/

1 Department of Architecture, Middle East Technical University, Ankara, Turkey 
volumetric relations, forms, performance etc. The literal definitions of mathematics and architecture clearly point out how these two disciplines intersect in the search for relations, references and space. Although this relationship is mostly perceived as a one way relationship, as mathematics in architecture, computational design paradigms and associated subjects like parametric models, generative systems, algorithmic thinking, and biomimesis emerging in the realm of computational design, architecture has also started to transform mathematics, and an "architecture of mathematics" has a new meaning that manifests this mutual relationship (Gönenç Sorguç 2012).

The computational design paradigm that compels algorithmic thinking requires a paradigmatic shift in architectural design education starting from the very first years. In this shift, the role of mathematics becomes more important than ever before. Pattern studies in general and tessellations specifically are promising subjects of mathematics which provide an invaluable mean to teach the fundamentals of computational design, allowing multi-dimensionality in relation with the complexity of the model, parametric models, generative systems and thus formations of patterns as a basis of algorithms to be discussed

In this paper, potentials of patterns first generated by regular/semi-regular tessellations and augmenting tessellations to multi-dimensions is discussed based on a series of explorations conducted in METU Mathematics in Architecture Class which aims to introduce students not only to mathematics in architecture but also computational design in architecture.

\section{The Role of Shape Studies in Developing Algorithmic Thinking}

The role of mathematics has always been acknowledged in the development of intellect and perception. Plato favoured mathematics as "a basis of philosophical thought" allowing us to understand relations which may not be physically demonstrated. Yet teaching mathematics has always been a challenge requiring mind blocks and math-phobia to be overcome, and thus it requires a continuous search for new means to communicate with learners.

The advances in computational technologies allowing designers to deal with complex systems requires a solid background in mathematics, and the role of mathematics has been re-defined in all the disciplines including architecture. Computational design as a new paradigm in architecture is not only changing design practice but also the position of the designer, who orchestrates and designs the process together with the end product design (artifice). The inevitable complexity of the design process resulting in complex computational models requires not only competence in mathematics (geometry, calculus etc.) but also the ability to develop algorithms and generative computational models to tackle complex multi-dimensional design problems.

An algorithm in simple terms can be defined as step-by-step instructions to solve a given problem for which the parameters, variables, constraints, objectives and resulting dimensionality should be clearly described (Gönenç Sorguç and Selçuk 2013). Here it is necessary to point out that dimensionality does not only mean 
spatial dimensions including time but also the number of parameters/variables constructing the computational model. No matter how complex the computational model/design and how unforeseeable the end product, the design process/algorithm should be a crystal box for the designer. This dilemma between the clarity of the design process and the unpredictability/complexity of the end product is one of the reasons for the changing role of the designer. Today, architectural design in the realm of computational technologies uses generative algorithms, complexity, system theories etc., and complex relationships and forces are manifested in the final form. It should be understood that the final form is composed of 3D linear or nonlinear geometries as the projection of these complex relationships stemming from dimensionality of the computational model. Therefore, shape studies, understanding patterns and the so-called "new mathematics in architecture" as discussed by Jane and Mark Burry (2012) gain a new importance in computational design. Understanding the complex geometries that result from the multi-dimensional computational design models and the relationships among them are essential in computational design practice.

This paradigmatic change forces designers also to find/improve the way these models are represented in such a way that the data/information/dimension shaping the computational models can be conveyed in the best possible way. Wilder and Chapman question "what makes a presentation or re-presentation of spatial relationships useful for thinking and for communicating effectively with others" and they discuss different ways of presenting three-dimensional objects in two dimensions (Johnston-Wilder and Mason 2005). The conventional architectural representation using sections and plans is a practice in which the information about higher dimensions is acquired by combining lower dimensions. However, complex multi-dimensional computational models of today's architecture demand a different practice to be constructed and/or represented. Consequently, today shape studies attain a new meaning in design education.

Shape is a vital, growing, and fascinating theme in mathematics with deep ties to classical geometry but goes far beyond it in content, meaning and method. Properly developed, the study of shape can form a central component of mathematics education, a component that draws on and contributes to not only mathematics but also to science and the arts (Marjorie Senechal quoted in Johnston-Wilder and Mason 2005)

In the realm of computational design education, shape and pattern studies offer great potentials to teach algorithmic thinking, generative systems etc. as discussed below.

Regular or semi-regular tessellations generated by symmetry groups developed by isometric transformations can be seen in architecture and art even in the very early examples in history and can be considered as rule-based systems regarding the generation processes. Any regular tessellation that can be decoded into the related symmetry group, and the primitive shapes generating the visual pattern i.e. the semantics and the syntax of the tessellating process, exemplifies the typical structure of a generative algorithm. Starting with a single motive ending with a complex two (three) dimensional shape organization with well-defined and formalized relations 
has a lot to learn from and offers an invaluable mean to further explore spatial relations of shapes, i.e. patterns and complex computational models.

Studies on tessellations and patterns are not new in art and architecture. Many examples of tessellations and patterns can be found in art and architecture from the very early examples either as ornaments or as a way of setting order to different systems and components. Various research studies in painting, architecture, music, dance, ceramics, carpeting, nature etc. have been conducted to decode these patterns in order to understand the generation/formation process and the constituents.

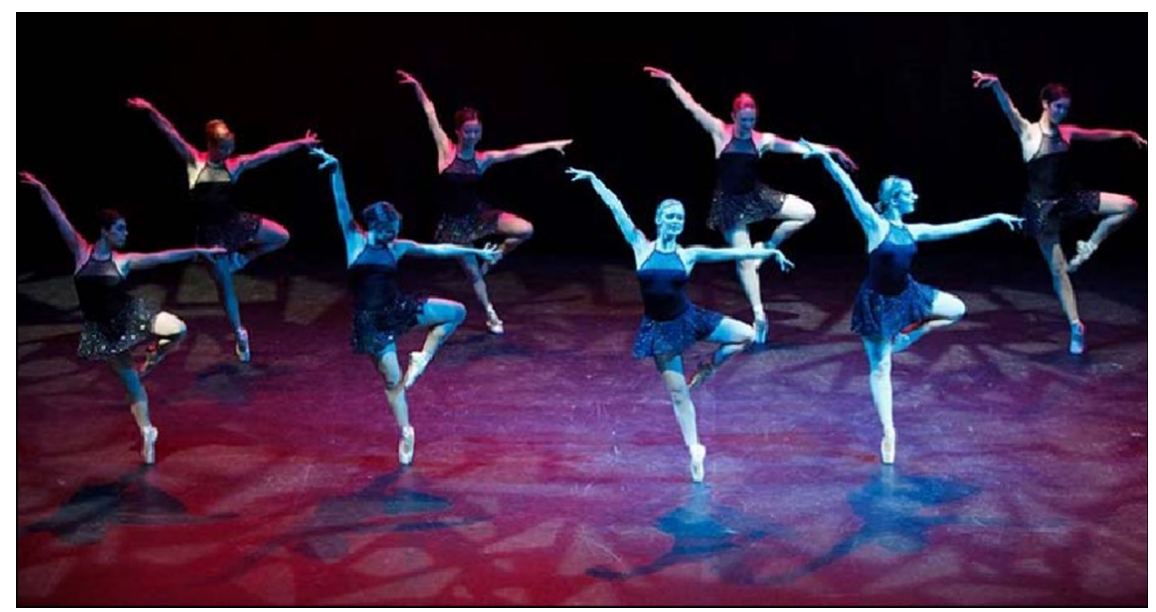

Fig. 1 Ballerinas in translation (Wasilewska 2012)
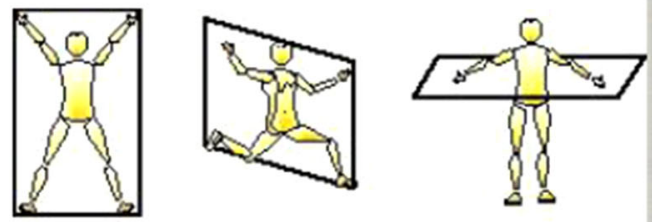

Rectangles haped Cartesian planes
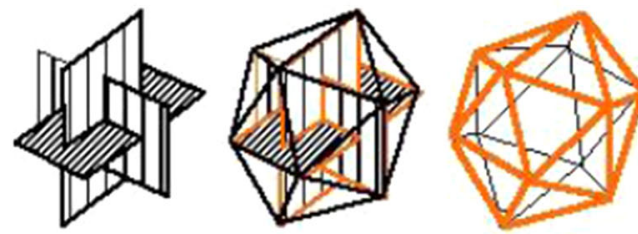

Linking thec orners of rectangular Cartesian planes builds an ic osahedral net.

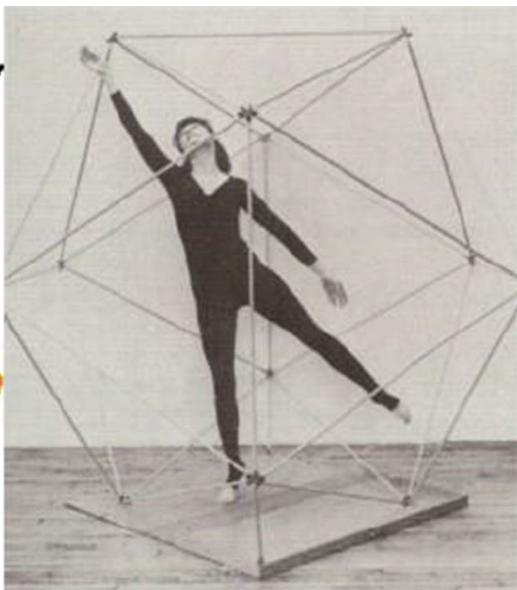

Fig. 2 Icosahedron reference system in Laban Notation (Corin 2000) 
Fig. 3 Laban notation (Corin 2000)
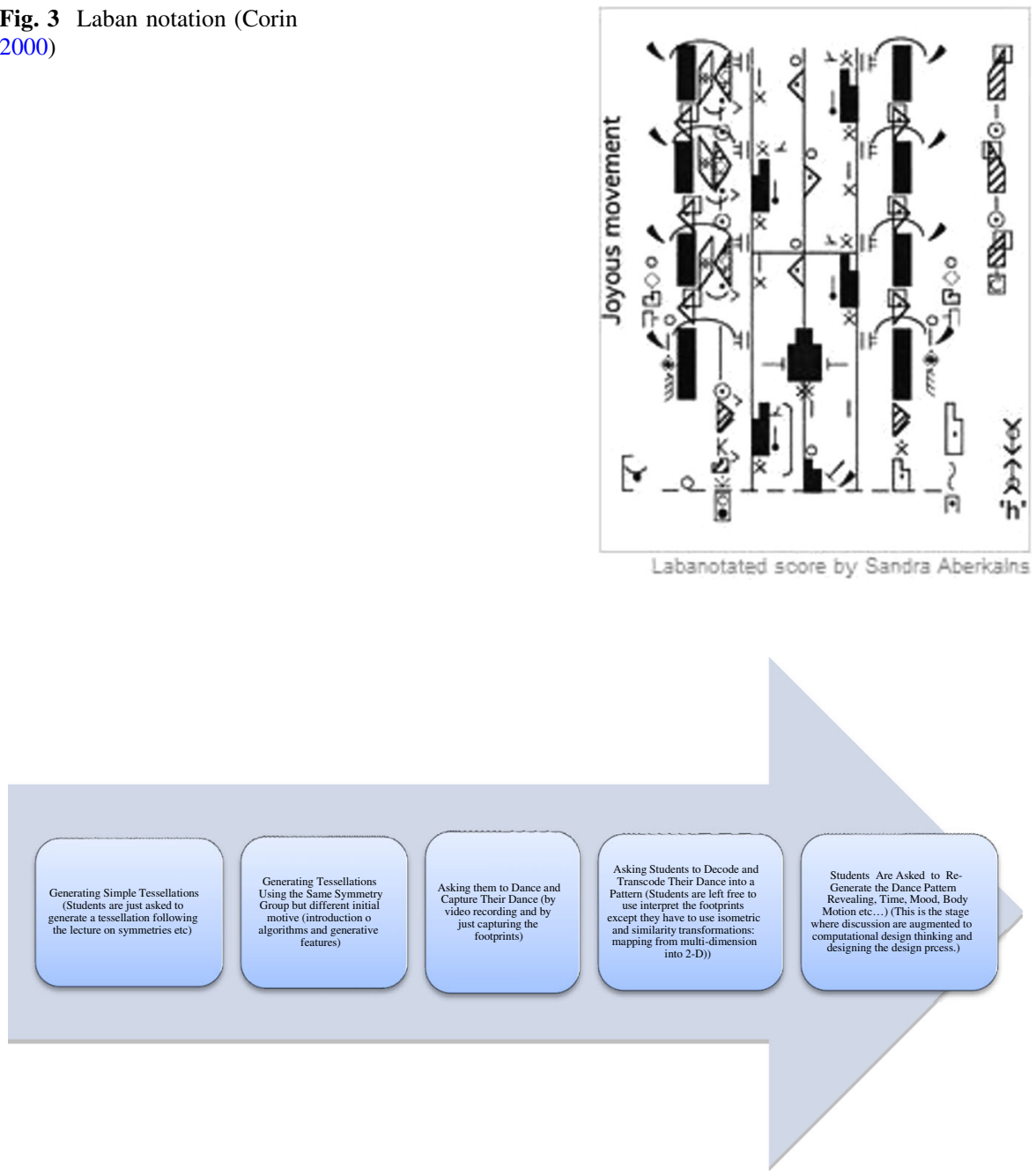

Fig. 4 The sequence of exploration in the course 'Mathematics in Architecture' (developed by Gönenç Sorguç 2015)

Dance, as one of the oldest acts/arts, has also some mathematics in its nature although these relationships are not as clear as in the other forms of art. There are a number of studies exploring the relationship between dance and mathematics or using mathematics as a way of understanding dance.

Wasilewska considers geometry as the most apparent field of mathematics present in dance (Wasilewska 2012):

We can consider the shapes, patterns, angles and symmetry of many different aspects of dance within a variety of scopes. The analysis could concern anything from one dancer frozen in a position to a whole ensemble actively moving in space. In the first case, we would look at the lines of the body and 
their relation to each other and to the space in which the dancer exists. In the latter, we would consider not only the lines and shapes created by the collective and the way in which they change with the music, but also the patterns of beats bringing on those changes.

Kelsey Danielle Siegel has tried to bridge between mathematics and modern dance:

Mathematics and modern dance, both uniquely defined languages and creative forms of communication, create a comprehensive understanding of physical space and our relationships to time, space, and energy. Their deep-rooted histories and specific trajectories give them the foundation needed in order to develop new ideas and enable perceptions of spatial relationships to evolve. As a compositional language, mathematics uses terms and definitions to serve as building blocks for abstract theorems and systems. Mathematics analyzes shapes and patterns in space with the use of symbols, values, variables, functions, and expressions. Modern dance, an embodied language, infuses these shapes and patterns in space within our bodies using various movement vocabularies, energies, and relations to space and one another (Siegel 2013).

Consequently, any dance which is "designed" by a strict choreography orchestrating the body movements and music in 3-D space can be considered as a spatial exploration in at least four dimensions (including time). How the dance patterns can be captured and transcoded into symbols has been a question for the choreographers and dancers requiring a very similar effort to design an algorithm/ system to design a multidimensional pattern. Laban notation or Kinetography Laban, as one of these recording systems first created by Rudolf Laban and then further developed, is a notation system for recording and analysing human movement in space including temporal changes employed not for dance but also in robotics, and human movement simulation (Dance Notation Bureau 2015). In this notation system, movement, duration and dynamic quality are transcoded into symbols where body and space has been related by an icosahedron reference rather than a Cartesian Reference system.

As can be seen in the notation in Figs. 1, 2, 3 and 4, transcoding the dance regarding the semantics and syntax, the resulting code (notation) is a very good example of how multi-dimensionality can be modelled in a much lower dimensional

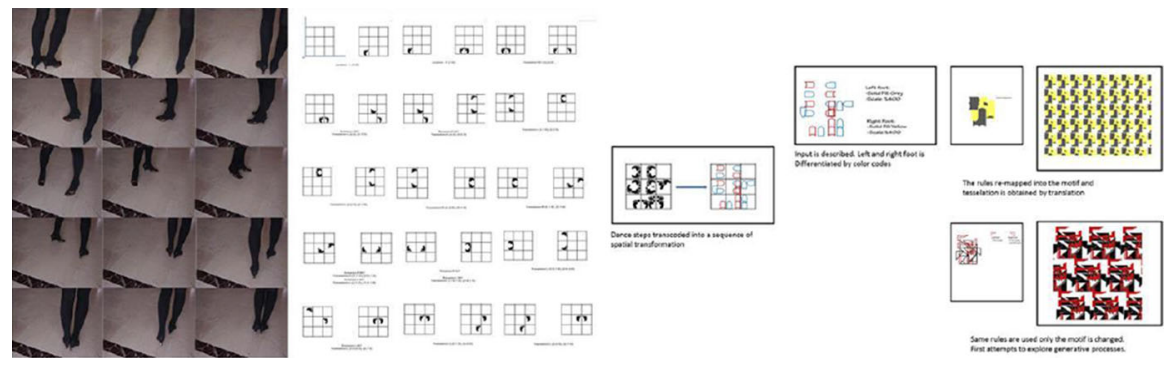

Fig. 5 Recording/decoding/generating: the dance machine (Günce Esingen, METU Arch) 

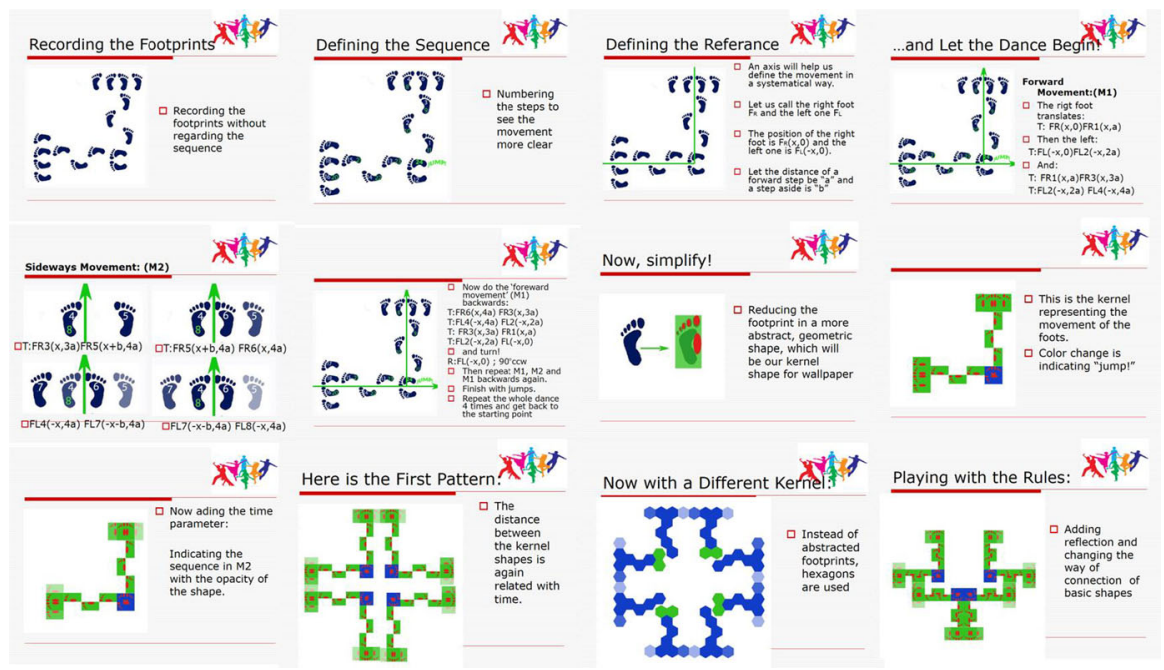

Fig. 6 Decoding the dance: need for a reference system, opacity and distance between the shapes as the mapping of time into 2-D (Burcu Karael, METU Arch)

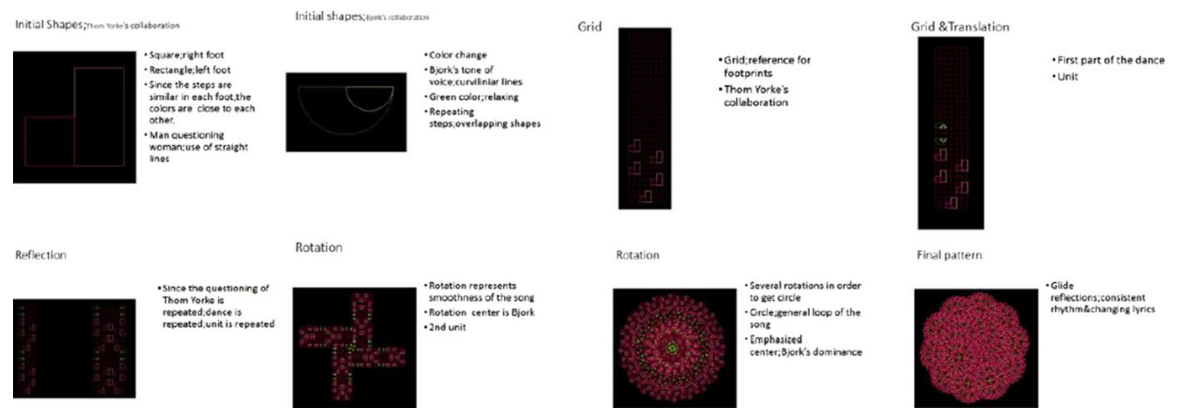

Fig. 7 Decoding the dance: mapping sound, emotions and time into 2-D patterns (Dicle Uzunyayla, METU Arch)

space (here in 2-D) without losing information as expected to be achieved in computational design models. Therefore, decoding the patterns of dance in space does not only provide potentials to study shapes and their spatial relations, but also provides a valuable means to discuss how multi-dimensional systems can be mapped one to another.

Hence, in this paper, the relationship between mathematics and dance is considered as a medium of teaching/learning computational design and algorithmic thinking starting from simple tessellations. Therefore the main focus of the present research is to show how these two different worlds: structured relations and studies in mathematics and the seemingly unstructured world of dance can serve to develop algorithmic thinking and an understanding of the nature of generative systems and multi-dimensionality in the realm of computational design. 

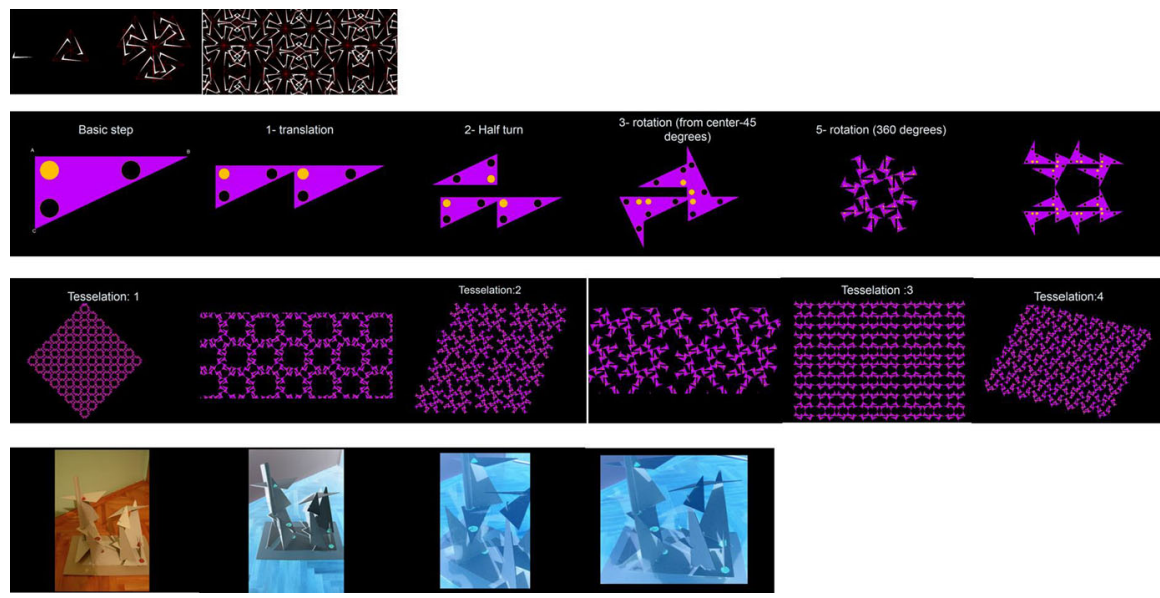

Fig. 8 First stage: a simple tessellation; Second stage: decoding the dance to obtain the primitive, tessellations by different symmetry groups-algorithm, trying to recover the lost dimension in 2-D spacemapping (Aslihan Günhan, METU Arch)
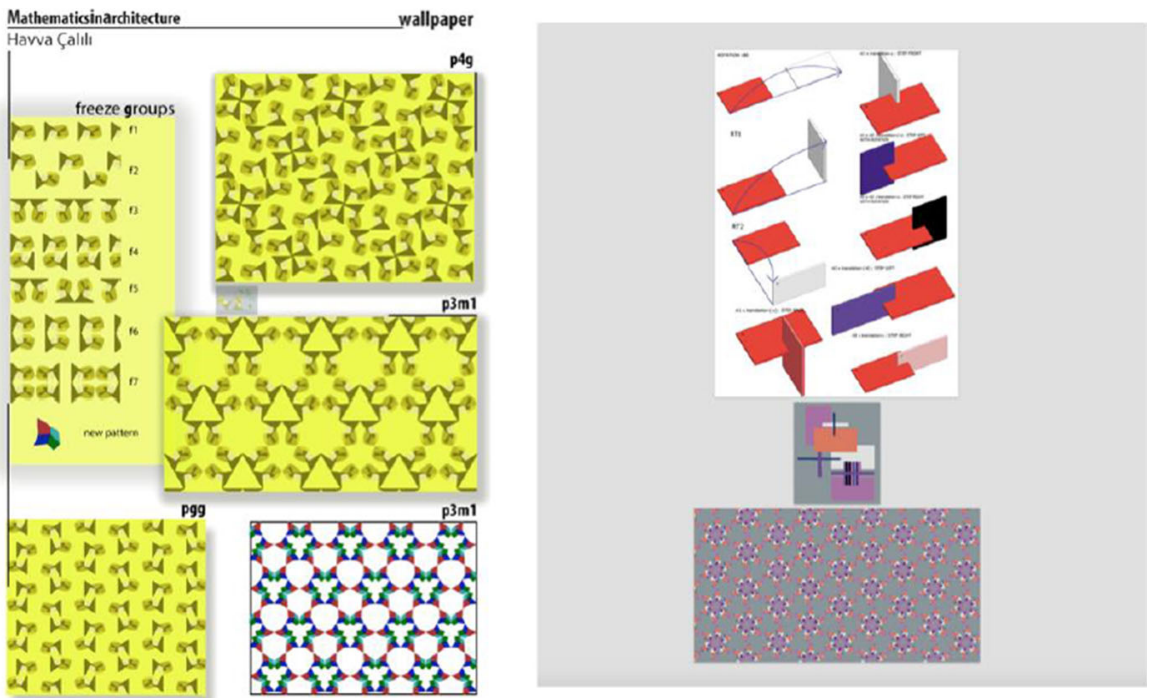

Fig. 9 First stage: a simple tessellation. Second stage: decoding the dance to obtain the primitive, tessellations by different symmetry groups-algorithm, trying to recover the lost dimension in 2-D space mapping (Havva Çalılı, METU Arch)

\section{Understanding What Pattern is in the Realm of Computation}

In computational design practice, it is essential to develop mathematical thinking skills in order to be capable of developing algorithms and processes of complex computational design models, necessitating new mindsets. One of the major challenges in this transformation is the development of algorithmic thinking 
entailing the definition of the design problems from ill-defined to well-defined, identification of all the parameters (variables, constraints, objectives to be achieved etc.) constructing the parametric model, describing/designing the "relations" and "patterns" and thus to design the design process. One of the definitions of pattern in the realm of computation is:

the formalization of a problem/solution pair, used to make an object-oriented design decision [What is Pattern? (undated)]

As can be seen in the definition, any pattern generation process can serve learners to understand the fundamentals of algorithmic thinking and computational design.

In this context, in the 'Mathematics of Architecture Arch 333' Course in METU [ARCH 333 (undated)], tessellations then patterns are re-visited as the first rulebased systems to introduce students to algorithmic thinking. These two-dimensional patterns generated by symmetry groups can also serve to understand "the idea of primitives" for further complex pattern generations. In the course of exploration, pattern generations are restricted by isometric and similarity transformations in order to avoid any confusion in the minds of students to understanding and following the thinking processes. The sequence of the exploration is illustrated in Figs. 5, 6, 7, 8 and 9 .

In order to extend the discussions to multi-dimensionality of complex models, dancing with their choreographies is then used as a new generative means for multidimensional patterns. Within the framework of the course students are asked to dance as they like and record their footprints as a projection of their choreography, which is multi-dimensional in nature. Decoding the footprints through isometric transformations in 2-D plane shows that the projection of the dance can also be modelled by one of (or a number of) 17 symmetry groups hence they can be used as the generative rules for new patterns. These new tessellations/patterns are then transposed as mappings from a multi-dimensional domain to two dimensional with a loss of information of some dimensions i.e. information. The next phase of the exercise is to explore how these missing (or lost dimensions) can be modelled in the pattern generation process, for which any relation between the domain and range can be set by the designer as in the case of computational design practice. The 'resulting tessellations/patterns', which are attempting to manifest higher

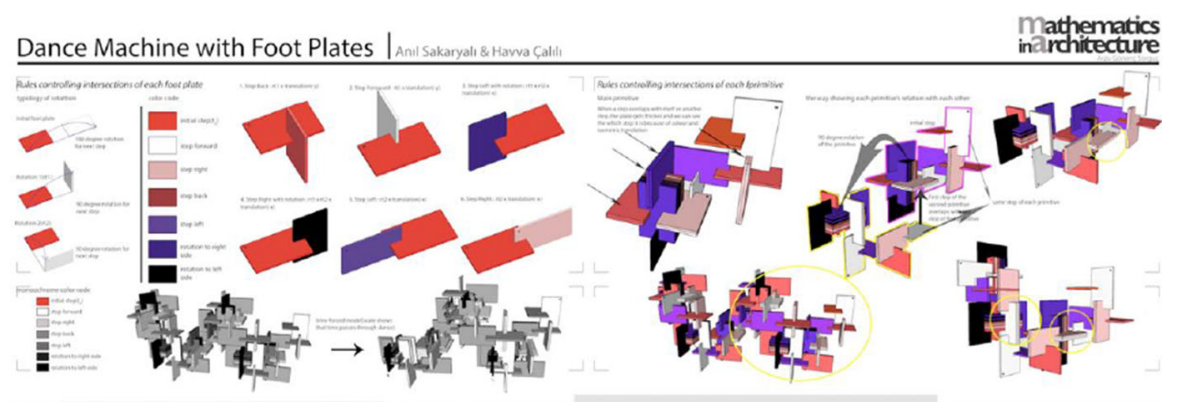

Fig. 10 Dance Machine (Havva Calıl1, Anıl Sakaryalı METU Arch) 


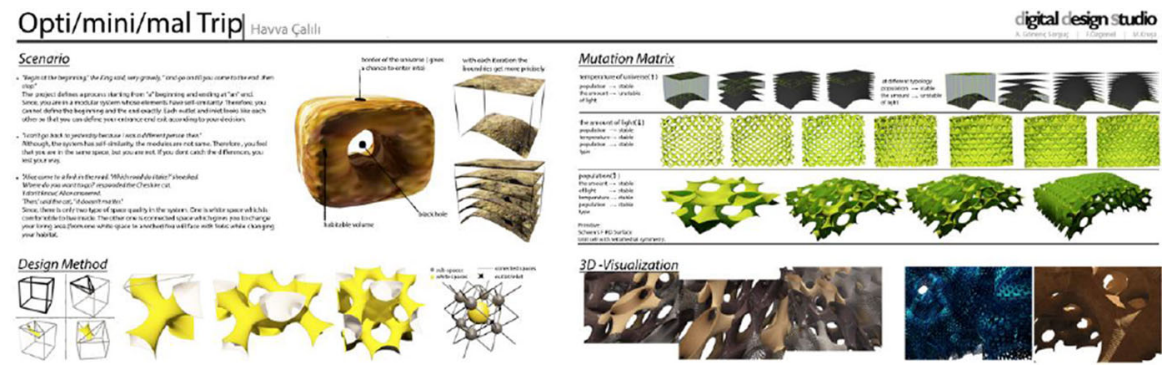

Fig. 11 Digital Design Studio Term Project (Havva Calılı METU Arch)

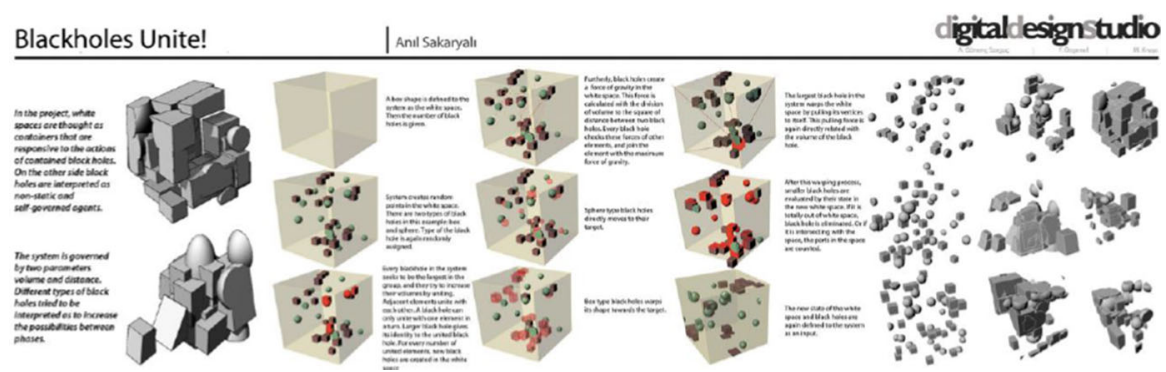

Fig. 12 Digital Design Studio Term Project (Anıl Sakaryalı METU Arch)

dimensions, is then studied for further discussion of computational design and algorithmic architecture.

In this study, how these discussions are carried out and how this exercise served for computational design teaching is explained by several student projects. Examples included here are selected randomly from the course database accumulated in eight years, in order to avoid any bias in explaining the process.

\section{The Exercise: Dance Machine}

This exercise is composed of three major steps and is usually assigned after completing the introduction to shape studies and study of symmetries, transformations and mapping. In the very first stage of the exercise students are just asked to generate wallpapers based on symmetry groups without being given any further information about the following steps; even the name of the exercise is not announced. At this point, students are concerned only about the aesthetic quality of their wallpaper and most of them relate this exercise to the basic design exercises given in the first year of their design education. In this phase of the exploration, critiques are only limited to figure-ground relations and the aesthetic quality of the wallpaper. Following this step, students are asked to explore possible variations first by changing the initial motive/primitive keeping the generation rule the same (in 

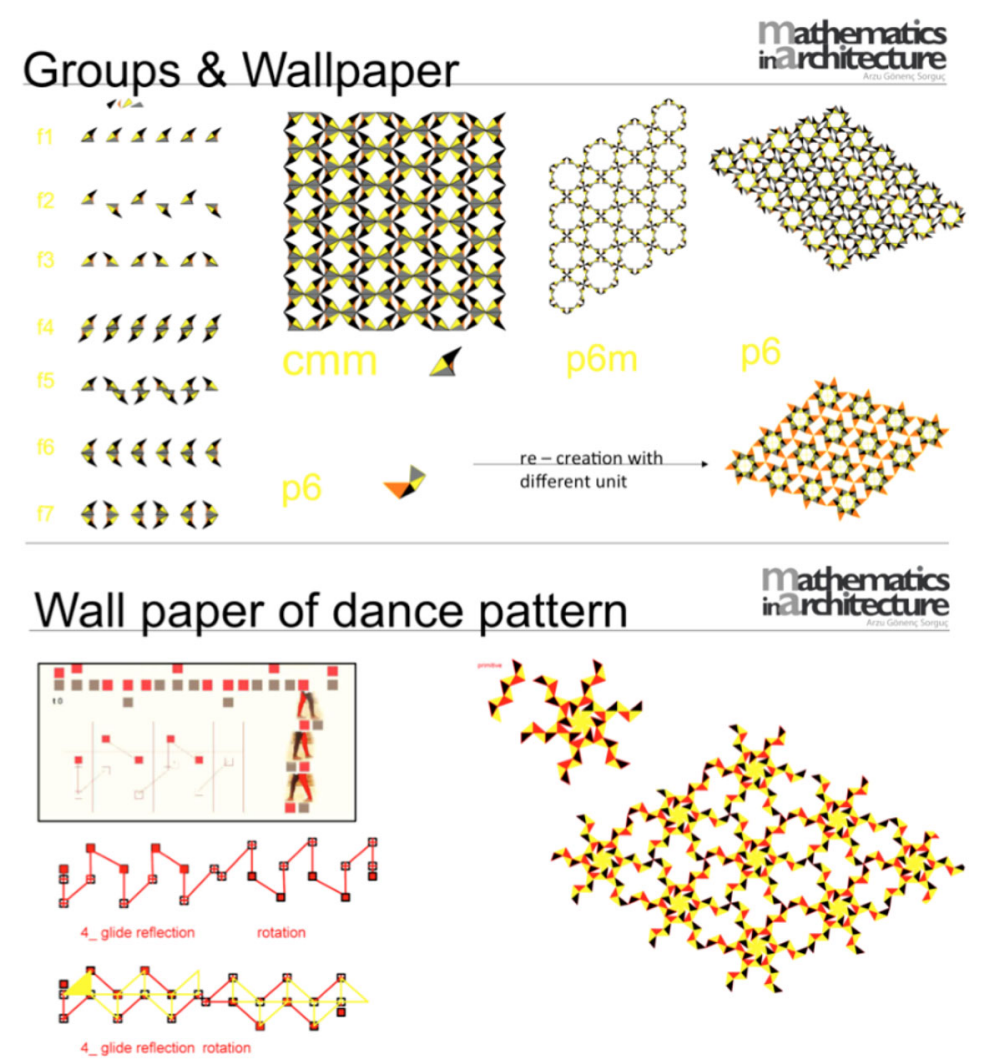

Fig. 13 First stage: a simple tessellation. Second stage: decoding the dance to obtain the primitive, tessellations by different symmetry groups-algorithm, trying to recover the lost dimension in 2-D space mapping (Asli Alp, METU Arch)

this case using the same symmetry group), as well as keeping the motive/primitive the same but changing the symmetry groups. This part of the exercise is a good leverage to break the mind block of students to the rule-based design as well as a medium to discuss the essential features of generative systems.

In the second stage, students are asked to dance as they like and no other explanation is given except to capture and then record their dance steps on 2-D plane. Then, they are asked to analyse their footprints and decode them as spatial transformations in plane by using simple transformations. Their experience in tessellations is revisited in this process. Once the decoding is achieved, students are re-introduced to the basic structure of algorithms and the idea of computational models and generative systems. Following these discussions, students are asked to re-generate/transcode tessellations based on the rules obtained through their decoding of dance patterns.

In this transcoding act, they are requested to consider the dimensions of the dance (not only spatial and temporal ones but also anything that they associated with their dancing act like mood, feelings, type of music and other dimensions that they 


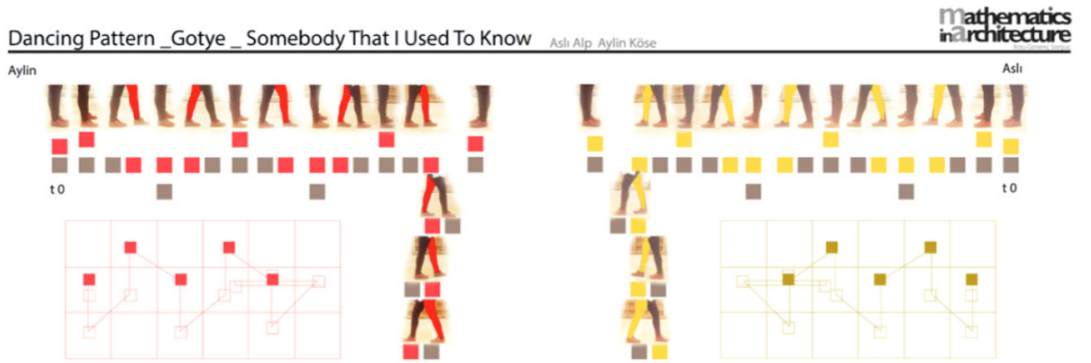

\section{Structure Of Rhytmic Patterns inarchitecture}
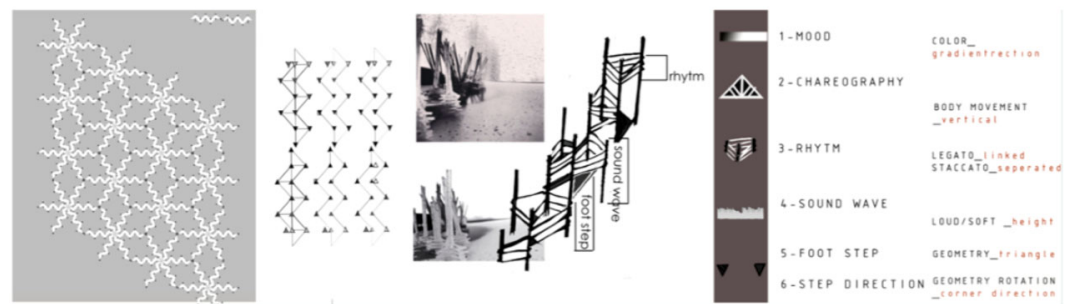

Fig. 14 Dance Machine (Aslı Alp, Aylin Köse METU Arch)

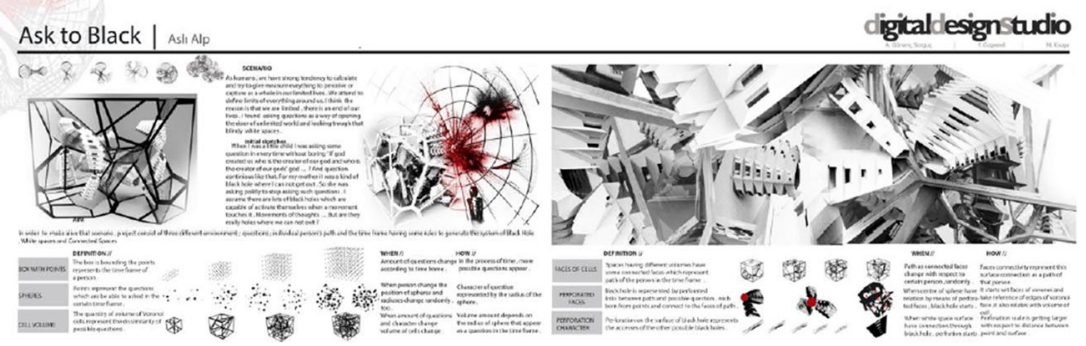

Fig. 15 Digital Design Studio Term Project (Asl1 Alp METU Arch)

identify) to advance discussions on multi-dimensionality in computational design and mapping from one domain to another as the basis of computational modelling.

They are expected to define their input (the primitive/motive) the rules (process/ algorithm) and to show the output (tessellations/patterns) and they are expected to model dimensions (information) other than the spatial ones.

Examples of classwork presented above show the differences on formalization of the process from one student to another. Yet, each example illustrates how students are analysing the domain, re-constructing the process and using it to generate another model. It is seen that students have attempted to map time, mood, body 
movements into the pattern and they set new relations between the domain (dance) and the range (pattern) by using scale, colour, transparencies, textures etc. Similar approaches are observed in all the studies completed in the course in last 7 years.

The examples that they generate in the very first stage of the exercise are compared with what they generate later by dance machine. This comparison provides a valuable opportunity to discuss what computational design is, what an algorithm is, and what a generative system means. One of the major paradigmatic shifts brought by computational design, "designing the process rather than the end product", has also been introduced and the role of mathematics has been emphasized once more.

In addition to the examples above, Figs. 10, 11, 12, 13, 14 and 15 show the classwork of METU Department of Architecture students Havva Çalılı (2014), Anıl Sakaryal1 (2014) and Aslı Alp who are attending Mathematics in Architecture Class and Digital Design Studio [ARCH 470 (undated)] in the same term. These are presented to give an idea about the progress of students in dealing with more complex computational design problems as shown in figures titled as "White Spaces, Black Holes: Connected Spaces". In years of experience of teaching Mathematics of Architecture Arch 333 (METU) and Digital Design Studio Arch 470 (METU) which is the first studio based on solely computational design, students who are taking mathematics class have adapted themselves to computational design more easily compared with other students who met with computational design practice for the first time. Moreover, these students stated that the way they approach the design problems is affected by algorithmic thinking and this has helped them in their design studios.

\section{Conclusion}

The potentials offered by computational technologies force us to have new mind sets which need to find ways to ease this shift in education and practice in any discipline. In this context, the role of mathematics has been admitted once more and it becomes the core of computational design practice, yet it is still one of the subjects that students fear. The paradoxical relationship between the importance of the subject and the math-phobia is one of the challenges to be overcome in computational design.

Hence, it is important to convince students that mathematics is a way of thinking and "it is everywhere". Dance serves well to achieve this goal. The "scary world of mathematics" and the "joyful world of dance" have been brought together in the dance machine in order to introduce computational design. The exercise shows that introducing mathematic in an unexpected medium such as dance helps students to re-position mathematics in their mind. It is also seen that basic mathematics will help students to understand much more complex relationships and modelling in architecture without any math phobia when this is overcome by tessellations and dance. 


\section{References}

ARCH 333 - METU Department of Architecture (undated) http://archweb.metu.edu.tr/programs/electivecourses/undergraduate-electives/arch-333 (accessed 20 January 2015).

ARCH 470 - METU Department of Architecture (undated) http://archweb.metu.edu.tr/programs/electivecourses/undergraduate-electives/arch-470 (accessed 20 January 2015).

Burry, J, and M. Burry. 2012. The New Mathematics in Architecture. London: Thames and Hudson.

Corin, Florence. 2000. Nouvelles De Danse $N^{\circ} 42-43$, Printemps- - Danse Et Architecture. Bruxelles: Contredanse.

Dance Notation Bureau. 2015. http://www.dancenotation.org (accessed 20 January 2015).

Gönenç Sorguç, A. 2012. Mathematics in Architecture, Architecture of Mathematics (Matematiğin Mimarlı̆̆ı, Mimarlıkta Matematik). Arredemento Mimarlık 256: 67-72.

Gönenç Sorguç, A., and S. Arslan Selçuk. 2013. Computational Models in Architecture: Understanding Muktidimensionality and Mapping. Nexus Mathematics in Architecture, Volume 15, Issue 2, pp 349-362, August 2013.

Johnston-Wilder, Sue and John Mason, eds. 2005. Developing Thinking in Geometry. London: Paul Chapman Publishing.

Siegel, Kelsey Danielle. 2013. Order and Chaos: Approaching Modern Dance Choreography in America Through a Mathematical Lens. Thesis, Wesleyan University Connecticut.

Wasilewska, Katarzyna. 2012. Mathematics in the World of Dance In: Proceedings of Bridges 2012: Mathematics, Music, Art, Architecture, Culture, eds Robert Bosch, Douglas McKenna and Reza Sarhangi, 453-456. Phoenix: Tessellations Publishing

What is Pattern? - A Word Definition From the Webopedia (undated) http://www.webopedia.com/TERM/ P/pattern.html (accessed 20 January 2015).

Arzu Gönenç Sorguç graduated from Middle East Technical University, Mechanical Engineering Department. She received her M.Sc. and Ph.D. degrees in Theory of Machines and Dynamic Systems from the Middle East Technical University, Mechanical Engineering Department as well. Since 1998, she has continued to work in the Department of Architecture as an Associate Professor in the same University. She is interested in acoustics non-standard structural design; sustainable and light weight structures, solid modeling and mesh enhancement, computational design and fabrication technologies, Biomimesis in architecture and origami. She became interested in Mathematics in Architecture in 1999 during her research into 'algorithmic thinking and the design process' and 'new tools for structural design'. Since 1998, she has conducted research at the Tokyo Institute of Technology, Department of Mechanical Sciences and Engineering, collaborating with Prof. Ichiro Hagiwara. 